\title{
Quantitative analysis of pungent principles of pepper oleoresins: Comparative study of three analytical methods
}

\author{
I. Noyer ${ }^{1}$, B. Fayet ${ }^{1}$, I. Pouliquen-Sonaglia ${ }^{2}$, M. Guerere ${ }^{1, *}$ and J. Lesgard ${ }^{2}$ \\ ${ }^{1}$ Laboratoire Interrégional de Marseille, Direction Générale de la Concurrence Consommation et Répression \\ des Fraudes, 146 traverse Charles Susini, 13388 Marseille Cedex 13, France \\ ${ }^{2}$ Faculté des Sciences et Techniques de Saint-Jérôme, Laboratoire de Chimie de Produits Naturels, \\ avenue Escadrille Normandie Niémen, 13397 Marseille Cedex 20, France
}

\begin{abstract}
For the determination of pungent compounds in pepper oleoresins, three analytical methods are described and compared to determine which of them is the most available for routine analysis and for informations about relative amount of all alkaloids. These methods are the UV spectrophotometry, the high-performance liquid-chromatography and the gas-chromatography. These analysis were carried out with 26 pepper oleoresins samples. The three methods were compared with two statistical tests, both defined in a French Norm from AFNOR. It seems that HPLC and GC methods, considering only the peak of piperine, are comparable. In the same way, HPLC and UV-spectrophotometry methods give similar results of total amount of alkaloids.
\end{abstract}

Key words. Oleoresin - piperine - UV spectrophotometry - high-performance liquid-chromatography (HPLC) - gas chromatography (GC).

\section{Introduction}

Pepper, from the seeds of Piper nigrum L., contains a variety of closely related nitrogen containing compounds, called alkaloids, and among which piperine (2-trans-4-trans- $N$ piperoyl-piperidine) is the main responsible of the characteristic pungent taste of pepper. All alkaloids are structurally related to piperine, but they may occur in very low concentration in pepper.

Pepper oleoresins are manufactured by extracting ground pepper with an organic solvent. They contain volatile and nonvolatile constituents which are extractable by the chosen solvent. Alkaloids represent $40-45 \%$ of the oleoresin composition.

Many of the older methods for the estimation of the pungency of pepper and its products measure all alkaloids collectively. These methods are polarographic, iodometric, spectrophotometric (especially UV) and colorimetric methods, or Kjeldahl determination of total nitrogen. The major inconvenient of these methods is their non-specificity.

Nevertheless pungent principles are still measured by UV spectrophotometry because it is rapid and cheap, and gives a good global estimation of the pungency [1,2]. It is the most used method by manufacturers.

However, there are circumstances in which a more specific determination of piperine is of value. Chromatographic methods, principally reversed-phase high-performance liquid chromatography (HPLC) and gas chromatography (GC) allow to separate alkaloids.

Already a number of HPLC methods for piperine have been reported [3-7]. They all used an UV-detector.

Nevertheless only some little studies report the analysis of piperine by gas chromatography $[8,9]$. This method requires chromatographic conditions with high oven temperature.

The present work describes a comparison of three methods: the spectrophotometry UV, the reversed-phase high-performance liquid chromatography (HPLC) and the gas chromatography (GC).

The aim of this study is to determine if a method, among the three, give more informations (i.e. amount of derived compounds and/or degradation state of piperine) than the two others, and, which method is the most available for routine analysis.

\footnotetext{
* Correspondence and reprints.
}

Received October 20, 1998; revised December 29, 1998; accepted January 06, 1999. 


\section{Original articles}

\section{Material and methods}

\section{Reagents}

All chemicals were of analytical reagent grade: ethanol 96\%, acetonitrile, acetic acid $1 \%$, pentane, dichloromethane. Piperine of purum grade $(98 \%)$ from Fluka. Hexacosane $\left(\mathrm{C}_{26}\right)$ 99\% from Fluka.

\section{Oleoresin samples}

26 pepper oleoresin samples were obtained from different suppliers of spices in France.

\section{Sample preparation}

\section{UV and HPLC}

$0.10 \mathrm{~g}$ of oleoresin were dissolved in $100 \mathrm{~mL}$ of ethanol $96 \%$. This solution was diluted 125-fold for UV and 5-fold for HPLC, in ethanol $96 \%$.

\section{GC}

$0.5 \mathrm{~g}$ of oleoresin were dissolved in $50 \mathrm{~mL}$ of dichloromethane. $2 \mathrm{~mL}$ of this oleoresin solution were added to $1 \mathrm{~mL}$ of internal standard solution (hexacosane $3 \mathrm{~g} / \mathrm{L}$ in pentane).

\section{Apparatus and methods}

\section{Spectrophotometry UV}

Spectrophotometric measurements were carried out on a UVIKON 992 (Kontron Instruments) spectrophotometer. Absorbances were measured in a $1 \mathrm{~cm}$ silica cell at $343 \mathrm{~nm}$ against the pure solvent as reference. Alkaloids were quantified by external standard method. Standard solutions were prepared by dissolving pure piperine in ethanol in concentration of 0.005 to $0.00125 \mathrm{~g} / \mathrm{L}$.

\section{HPLC}

The equipment used was as follows: a WATERS chromatograph equipped with a pump (WATERS 600E); a diode array detector with a wavelength field from 190 to $800 \mathrm{~nm}$ (WATERS 990 SERIES). Analyses were carried out on a ready-packed column $(250 \times 4.6 \mathrm{~mm})$ of $5 \mu \mathrm{m}$ Nucleosil $120 \mathrm{~A}$ (a fully-capped $\mathrm{C}_{18}$ bonded-phase). The mobile phase was a mixture of $48 \%(\mathrm{v})$ acetonitrile - $52 \%(\mathrm{v})$ water $(1 \%$ acetic acid). The flow rate was $1 \mathrm{~mL} / \mathrm{min}$ and the injection volume $40 \mu \mathrm{L}$. All analyses were carried out at four wavelengths, 254-280-343 and $364 \mathrm{~nm}$. Run time was up to 45 min to allow elution of all the minor UV-absorbers, and piperine retention time was c.a. 20 min. Piperine was quantified by external standard method. Standard solutions were prepared by dissolving pure piperine in ethanol in concentration of 0.10 to $0.025 \mathrm{~g} / \mathrm{L}$. Alkaloids were quantified assimilating them to the piperine and utilizing the same standard solutions than for the piperine quantification.

\section{Gas chromatography}

The equipment used was a PERKIN ELMER Autosystem chromatograph equipped with a FID detector. The GC analysis were performed on a capillary apolar column BP1 (50 $\mathrm{m} \times 0.22 \mathrm{~mm}$ I.D., $0.25 \mu \mathrm{m}$ filmthickness). The operating parameters were as follows: hydrogen as carrier gas at $1 \mathrm{~mL} / \mathrm{min}$, split at $25 \mathrm{~mL} / \mathrm{min}$, injector temperature at $300{ }^{\circ} \mathrm{C}$, detector temperature at $300{ }^{\circ} \mathrm{C}$, and following program conditions: $250{ }^{\circ} \mathrm{C}$ to $280{ }^{\circ} \mathrm{C}$ at $0.5{ }^{\circ} \mathrm{C} / \mathrm{min}$. Run time was up to $35 \mathrm{~min}$, piperine retention time was c.a. $20 \mathrm{~min}$ and hexacosane retention time c.a. 13 min. Alkaloids were quantified by internal standard method. The internal standard is hexacosane. The standard solution is prepared by adding $2 \mathrm{~mL}$ of pure piperine solution (4 g/L in dichloromethane) to $1 \mathrm{~mL}$ of hexacosane solution $(3 \mathrm{~g} / \mathrm{L}$ in pentane).

\section{Results and discussion}

Before determining piperine contents in oleoresin samples, the variation coefficient was calculated for each method and was found to be:

\section{$0.26 \%$ for UV-spectrophotometry; \\ $1.73 \%$ for HPLC; \\ $2.87 \%$ for GC.}

The piperine and/or alkaloids contents were determined for each sample by using the three methods described above.

In HPLC analysis, the four wavelengths (254-280-343 and $364 \mathrm{~nm}$ ) were chosen because 254 and $280 \mathrm{~nm}$ are commonly available in fixed-wavelength detectors, and 343 and $364 \mathrm{~nm}$ represent the absorption maxima of piperine and piperettine (the major derived compound of piperine) respectively.

For each wavelength, the piperine content (noted "HPLC") was determined and for $\lambda=343 \mathrm{~nm}$ it was calculated also a "total alkaloids" content (noted "HPLCt"), by considering the whole peak of the chromatogram and by assimilating it to piperine and supposing that they have all the same molecular extinction coefficient $\varepsilon$. (Tab. I).

In GC analysis, similar determinations were performed: piperine content (noted "GC") and "total alkaloids" content (noted "GCt") by supposing that they have all the same response factor K. (Tab. I).

\section{Comparison of the HPLC measures at four wavelenghts (254-280-343-364 nm)}

To improve the comparisons a variance analysis was carried out with 3 samples (sample 2, sample 11 and sample 18) among the 26. These three samples were chosen as explained: the two extreme values of piperine amount and a middle value.

The variance analysis is a comparison between different means in order to show effects due to the variation of experimental factors which can't be confused with the noise. 


\section{Original articles}

Table I. Results (\%) of UV, HPLC and GC analysis.

\begin{tabular}{|c|c|c|c|c|c|c|c|c|}
\hline \multirow[t]{2}{*}{$\begin{array}{l}\text { Sample } \\
\text { number }\end{array}$} & \multirow{2}{*}{$\begin{array}{l}U V(\%) \\
343 \mathrm{~nm}\end{array}$} & \multicolumn{4}{|c|}{$\begin{array}{l}\text { HPLC (\%) } \\
\text { (only piperine) }\end{array}$} & \multirow{2}{*}{$\begin{array}{c}\text { HPLCt }(\%) \\
\text { (all alkaloids) } \\
343 \mathrm{~nm}\end{array}$} & \multirow[t]{2}{*}{$\begin{array}{c}G C(\%) \\
\text { (only piperine) }\end{array}$} & \multirow[t]{2}{*}{$\begin{array}{c}\text { GCt }(\%) \\
\text { (all alkaloids }\end{array}$} \\
\hline & & $254 \mathrm{~nm}$ & $280 \mathrm{~nm}$ & $343 \mathrm{~nm}$ & $364 \mathrm{~nm}$ & & & \\
\hline 1 & 52.71 & 42.35 & 42.29 & 45.61 & 42.31 & 52.49 & 46.07 & 48.88 \\
\hline 2 & 23.88 & 10.77 & 10.78 & 14.62 & 10.72 & 23.60 & 14.80 & 23.01 \\
\hline 3 & 45.09 & 38.25 & 38.12 & 38.53 & 38.17 & 44.92 & 38.49 & 43.68 \\
\hline 4 & 36.74 & 26.94 & 26.73 & 27.11 & 26.75 & 36.69 & 27.15 & 35.48 \\
\hline 5 & 35.77 & 27.53 & 27.61 & 27.79 & 27.51 & 35.58 & 28.06 & 33.49 \\
\hline 6 & 44.98 & 36.98 & 36.88 & 37.45 & 36.90 & 44.70 & 38.84 & 46.29 \\
\hline 7 & 42.26 & 34.05 & 34.03 & 35.65 & 34.00 & 42.08 & 36.78 & 42.48 \\
\hline 8 & 42.25 & 33.65 & 33.57 & 35.63 & 33.57 & 42.15 & 36.34 & 40.77 \\
\hline 9 & 45.69 & 38.18 & 38.31 & 39.04 & 38.35 & 45.99 & 39.37 & 48.77 \\
\hline 10 & 36.15 & 30.21 & 30.26 & 30.59 & 30.22 & 36.78 & 30.46 & 34.86 \\
\hline 11 & 52.41 & 38.00 & 38.11 & 45.03 & 38.14 & 52.62 & 44.93 & 55.08 \\
\hline 12 & 55.14 & 41.96 & 42.05 & 48.43 & 42.02 & 55.14 & 47.69 & 50.12 \\
\hline 13 & 49.25 & 42.21 & 42.25 & 42.47 & 42.18 & 49.30 & 42.19 & 43.54 \\
\hline 14 & 47.24 & 38.53 & 38.62 & 38.98 & 38.64 & 47.17 & 39.06 & 40.12 \\
\hline 15 & 44.33 & 36.24 & 36.25 & 37.16 & 36.21 & 44.17 & 37.27 & 48.36 \\
\hline 16 & 35.00 & 26.80 & 26.71 & 26.97 & 26.71 & 34.66 & 27.54 & 32.64 \\
\hline 17 & 40.64 & 30.53 & 30.33 & 32.57 & 30.65 & 40.33 & 32.62 & 33.92 \\
\hline 18 & 54.61 & 50.23 & 50.21 & 50.21 & 50.18 & 54.52 & 50.02 & 51.11 \\
\hline 19 & 41.11 & 32.98 & 32.94 & 34.18 & 32.97 & 40.72 & 34.72 & 39.67 \\
\hline 20 & 45.10 & 37.11 & 37.06 & 38.28 & 37.16 & 45.08 & 38.56 & 42.87 \\
\hline 21 & 43.61 & 36.06 & 36.16 & 37.64 & 36.30 & 43.53 & 38.30 & 45.46 \\
\hline 22 & 46.27 & 35.27 & 35.24 & 39.07 & 35.28 & 46.45 & 39.80 & 59.06 \\
\hline 23 & 40.15 & 32.50 & 32.61 & 32.95 & 32.60 & 39.88 & 33.07 & 44.83 \\
\hline 24 & 52.64 & 40.92 & 40.95 & 45.45 & 41.21 & 52.80 & 44.61 & 58.55 \\
\hline 25 & 47.67 & 35.42 & 35.39 & 38.99 & 35.39 & 47.84 & 39.68 & 58.14 \\
\hline 26 & 46.53 & 38.54 & 38.39 & 39.82 & 38.51 & 46.15 & 40.66 & 46.05 \\
\hline
\end{tabular}

Two variances are calculated: the variance "inter-samples" $\left(s^{2}{ }_{\text {inter }}\right)$ and the variance "intra-samples" $\left(s^{2}{ }_{\text {intra }}\right)$.

Then a test of Fisher is carried out. It consists to compare the experimental $F$ value $\left(F_{\text {exp }}\right)$ obtained by the ratio $s^{2}{ }_{\text {inter }} / s^{2}{ }_{\text {intra }}$ with the theorical $F$ value $\left(F_{\text {th }}\right)$ given in a Fisher table:

if $F_{\text {exp }}>F_{\text {th }}$, the effect of experimental factors is more important than the effect of random errors due to the noise,

if $F_{\text {exp }}<F_{\text {th }}$ the effect of experimental factors is not significatively different from the effect of random errors.

In our case, the variance "inter-samples" corresponds at the variance between the four wavelengths and the variance "intra-samples" corresponds at the variance between the six injections of the sample at the same wavelength. The calculations give:

- for sample 2 :

$s_{\text {inter }}^{2}=0.040$
$s^{2}{ }_{\text {intra }}=0.090$
$F_{\exp }=0.444$

$F_{\text {th }}=3.098$

- for sample 11:

$s^{2}{ }_{\text {inter }}=0.022$

$s_{\text {intra }}^{2}=0.011$

$F_{\text {exp }}=2.000$

$F_{\text {th }}=3.098$

- for sample 18:

$s^{2}{ }_{\text {inter }}=0.019$

$s_{\text {intra }}^{2}=0.026$

$F_{\text {exp }}=0.731$

$F_{\text {th }}=3.098$.

In each case, $F_{\exp }<F_{\text {th }}$. Then, for the three samples, the variance analysis showed that, for a 5\% error of first kind, the effect of experimental factors is not significatively different from the effect of random errors. It means that the piperine content was found to be the same whatever the chosen wavelength. 


\section{Original articles}

This conclusion achieved with only three samples can be extrapolated to the entire of the 26 samples, taking into account the way of these three samples choise.

This comparison have already been reported [5]. It was carried out with 3 ground black peppers, 1 ground white pepper and only 3 pepper oleoresins. In our present study, it is confirmed by analyzing 26 oleoresin samples and by a variance analysis.

These results show that piperine contents obtained at 254-280-343 or $364 \mathrm{~nm}$ were similar and would probably be suitable for routine use. So that laboratories equipped only with a fixed-wavelength UV-detector should be able to use this HPLC method.

\section{Comparison of the three methods (spectrophotometry UV, HPLC and GC)}

Comparison were carried out by two statistical tests defined both in a French Norm.

One used the NF X 06-066 standard based on the comparison of paired observations [10]. The initial null hypothesis is that the two methods give identical results, considering sampling errors and random errors of measures. The used test is based on the comparison between the mean of differences population and a gived mean at 0 . For a $5 \%$ error of first kind, the inegality is verified:

$$
\left|d_{\text {mean }}\right|<t_{\left(1-\frac{\alpha}{2}, v=n-1\right)} \frac{S_{d}}{\sqrt{n}}
$$

with $\left|d_{\text {mean }}\right|$ : absolute value of differences $(d)$ mean.

and

$$
S_{d}=\frac{1}{n-1} \sum d^{2}-\frac{1}{n}\left(\sum d\right)^{2}
$$

with $n$ : amount of paired measures

$d$ : differences between the two series of measures

$t$ : fractile of Student test to accept the null hypothesis.

The second used statistical test, described in the NF V 03110 standard, is based on the protocol for evaluation of an alternative quantitative method against a reference method [11]. The relation between the alternative method and the reference method is evaluated by the linear equation $Y=b X$ $+a$. The two methods are considered without systematic bias if this equation is not significatively different of $Y=X$. That is confirmed by the two inegalities:

for the slope:

$$
b-t_{\left(1-\frac{\alpha}{2}, v=n-2\right)} \times S_{b}<1<b+t_{\left(1-\frac{\alpha}{2}, v=n-2\right)} \times S_{b}
$$

for the origin $Y$-axis:

$$
d_{\text {mean }}-t_{\left(1-\frac{\alpha}{2}, v=n-1\right)} \times \frac{S_{d}}{\sqrt{n}}<0<d_{\text {mean }}-t_{\left(1-\frac{\alpha}{2}, v=n-1\right)} \times \frac{S_{d}}{\sqrt{n}}
$$

with: $d_{\text {mean }}$ : mean of the differences $d$;

$S_{b}$ : standard deviation of the slope $b$;

$S_{d}$ : standard deviation of the differences $d$.

\section{Comparison of the HPLC and the UV results}

For this comparison all the peaks present on the HPLC chromatogram should be considered and the UV-detection was carried out at $343 \mathrm{~nm}$ (Tab. I, Fig. 1).

The two series of measures were compared by the two statistical tests defined above.

For the first one [10], calculations for the comparison give:

$$
\begin{aligned}
& d_{\text {mean }}=-0.07 \\
& \begin{array}{l}
S_{d}=0.24 \\
n=26 \quad \mathrm{v}=
\end{array}-1=25 \quad 1-\alpha / 2=0.975 \quad t=2.060 \\
& t_{\left(1-\frac{\alpha}{2}, v=n-1\right)} \times \frac{S_{d}}{\sqrt{n}}=0.10 .
\end{aligned}
$$

The inegality (1) is respected, then the two methods (HPLC and UV at $343 \mathrm{~nm}$ ) give similar results, with a 5\% error of first kind.

For the second one [11], the calculations give the linear equation $Y=0.99 X+0.51$ and the intervals are $[0.95,1.03]$ for the slope a and $[-0.21,0.35]$ for the initial $Y$-axis.

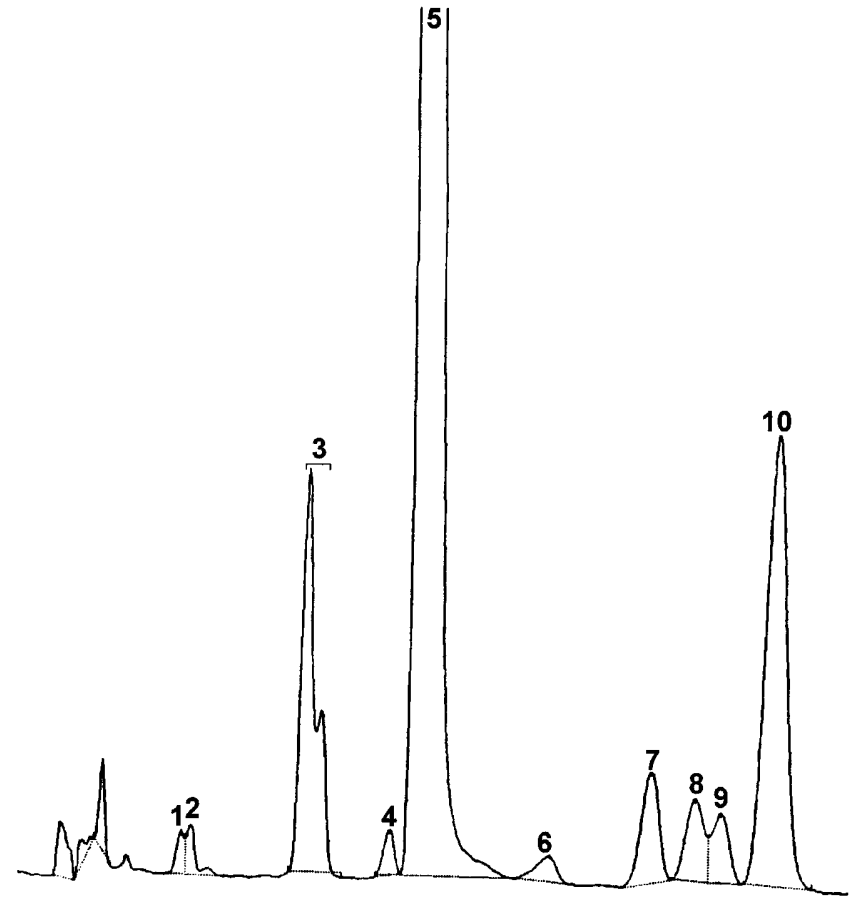

Fig. 1. HPLC chromatogram of a pepper oleoresin, at $343 \mathrm{~nm} .1$ : unidentified peak, 2: unidentified peak, 3: piperlonguminine + piperyline, 4: piperine photoisomers, 5: piperine, 6: unidentified peak, 7: piperettyline, 8: piperoleine A, 9: piperoleine B, 10: piperettine. 
The two inegalities, (2) and (3), are respected, then the two methods didn't show a systematic bias.

The two statistical tests were verified, then the HPLC method and the UV method are comparable.

It means that the quantitative measurement of total pungency in oleoresin can be carried out indifferently by HPLC or UV method, at $343 \mathrm{~nm}$. Both give a good estimation of the global $343 \mathrm{~nm}-\mathrm{UV}$-absorber compounds contained in the pepper oleoresins. Though the UV method is not specific, it is faster than the HPLC method. So it is preferred by manufacturers.

\section{Comparison of HPLC and GC methods considering only the piperine}

For this comparison only the peak of piperine on the HPLC and on the GC chromatograms is taken into account (Tab. I, Figs. 1 and 2). Furthermore HPLC measures were carried out at $343 \mathrm{~nm}$, which is the absorption maximum of piperine.

The two series of measures were compared by the two statistical tests defined above.

For the first one [10], calculations for the comparison give:

$$
\begin{aligned}
& d_{\text {mean }}=-0,26 . \\
& S_{d}=1.77 \\
& n=26 \quad 1-\alpha / 2=0.975 \quad t=2.060
\end{aligned}
$$

$$
t_{\left(1-\frac{\alpha}{2}, v=n-1\right)} \times \frac{S_{d}}{\sqrt{n}}=0.72 .
$$

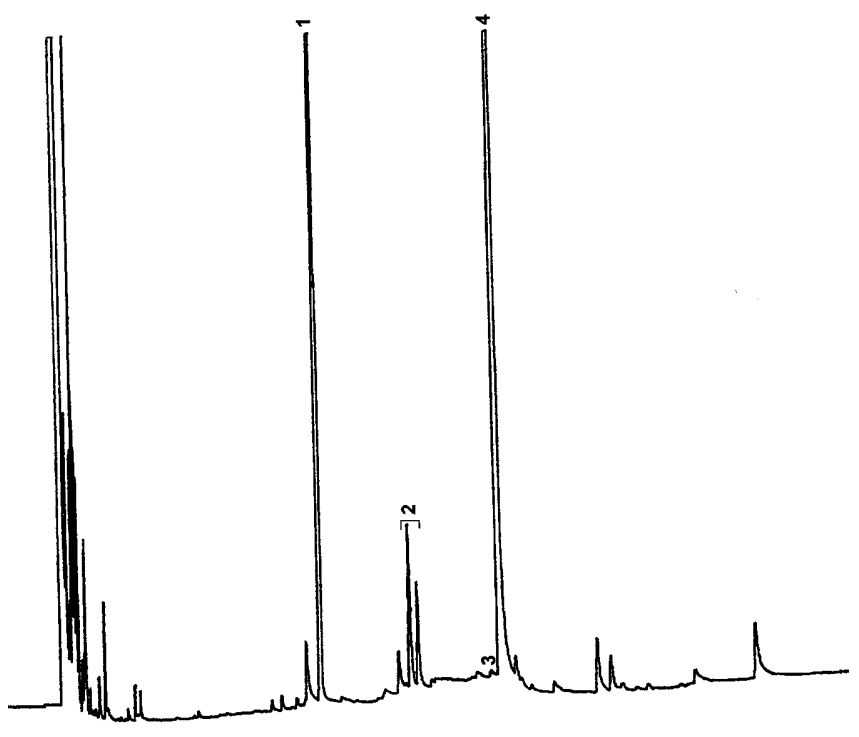

Fig. 2. GC chromatogram of a pepper oleoresin, 1: internal standard, 2: piperine photoisomers, 3: alkaloids, 4: piperine + alkaloids.
The inegality (1) is respected, then the two methods (HPLC at $343 \mathrm{~nm}$ and GC) give similar results, with a 5\% error of first kind.

For the second one [11], the calculations give the linear equation $Y=1.01 X-0.76$ and the intervals are $[0.98,1.04]$ for the slope a and [0.06, 0.47] for the initial $Y$-axis. The inegality for the slope (2) is respected but the inegality for the initial $Y$-axis (3) isn't respected. It means that the two methods showed a systematic bias. Indeed by observing the two series of measures, it can be observed that the GC method overvalued lightly results.

However the differences between GC and HPLC measures are included in intervals of the variation coefficients of the two methods. Furthermore the first statistical test [10] show that the two methods give similar results. It is so possible to consider that results obtained by HPLC and those obtained by GC are comparable. Though the measures are lightly majored by the GC method, it is faster than HPLC method because it requires a run time c.a. 20 min against $45 \mathrm{~min}$ by HPLC.

\section{Comparison of UV spectrophotometry - GC methods and HPLC - GC methods, considering all the alkaloids}

For these comparisons all the peaks present on the two chromatograms (HPLC and GC) were examined (Tab. I, Figs. 1 and 2). The UV measures were carried out at $343 \mathrm{~nm}$.

For the comparison between UV-spectrophotometry and GC methods, the results of the two statistical tests were gived. give:

- for the first one [10], calculations for the comparison

$$
\begin{aligned}
& d_{\text {mean }}=0.00 \\
& S_{\mathrm{d}}=4.79 \\
& n=26 \quad 1-\alpha / 2=0.975 \quad t=2.060 \\
& t_{\left(1-\frac{\alpha}{2}, v=n-1\right)} \times \frac{S_{d}}{\sqrt{n}}=1.94 \text {. }
\end{aligned}
$$

The inegality (1) is respected, then the two methods (UV at $343 \mathrm{~nm}$ and GC) give similar results, with a 5\% error of first kind.

- for the second one [11], the calculations give the linear equation $Y=0.67 X-14.56$ and the intervals are [0.48, $0.86]$ for the slope a and $[-2.88,2.08]$ for the initial Y-axis. The inegality for the slope (2) is not respected but the inegality for the initial $Y$-axis (3) is respected. It means that the two methods showed a systematic bias.

For the comparison between HPLC and GC methods, the results of the two statistical tests were given. give:

- for the first one [10], calculations for the comparison

$d_{\text {mean }}=0.07$

$S_{d}=4.72$ 


\section{Original articles}

$$
\begin{gathered}
n=26 \quad v=n-1=25 \quad 1-\alpha / 2=0.975 \quad t=2.060 \\
t_{\left(1-\frac{\alpha}{2}, v=n-1\right)} \times \frac{S_{d}}{\sqrt{n}}=1.91 .
\end{gathered}
$$

The inegality (1) is respected, then the two methods (HPLC at $343 \mathrm{~nm}$ and GC) give similar results, with a $5 \%$ error of first kind.

- for the second one [11], the calculations give the linear equation $Y=0.68 X-13.92$ and the intervals are $[0.49$, $0.87]$ for the slope a and $[-1.98,1.83]$ for the initial $Y$-axis. The inegality for the slope (2) is not respected but the inegality for the initial $Y$-axis (3) is respected. It means that the two methods showed a systematic bias.

This systematic bias between GC and UV methods (spectrophotometry and HPLC) can be explain differently.

It has been shown that UV-spectrophotometry and HPLC methods give similar results. At $343 \mathrm{~nm}$, on the HPLC chromatogram we can see 10 peaks. Their identification shows that they are all alkaloids. So that proves that the UV analysis (spectrophotometry and HPLC) at $343 \mathrm{~nm}$ measure only the alcaloids and no other compounds.

In UV measures, all compounds don't have the same extinction coefficient $(\varepsilon)$, so they don't have the same UV response. Then the quantitation is erroneous because they are all assimilated to the piperine. Nevertheless, in CPG, according to their very similar structure, we can suppose that the alcaloids have all approximatively the same response factor $(K)$. So it is possible to assimilate all of them to piperine without carrying out a significative error about the quantitative result.

However the GC chromatogram shows more peaks than the HPLC one. On the GC chromatogram, some peaks represent alkaloids but the other peaks represent probably other compounds present in pepper oleoresins. The amount of these other compounds is more or less important depending on the oleoresin. The variations of "non alkaloids" quantities seem to be in relation with the differences between the HPLC measures and the GC measures.

So, the different methods have all advantages and inconvenients, and it is difficult to choose one.

The photoisomers of piperine (i.e. compounds obtained by exposing piperine to light) are best separated by GC than by HPLC, but the derived compounds of piperine are well separated by HPLC. So, it appears that GC analysis give more information than HPLC about the degradation state of piperine, but HPLC gives more information than GC about the amount of alkaloids against piperine.
It is known that the photoisomerisation of the piperine decreases the pungency of the product [12]. But what influence of the pungency have the more or less important proportion of alkaloids versus the amount of piperine? Any data were given in the litterature about that. To examine the influence of all the alkaloids on the pungency of pepper, it may be interesting to carry out taste analysis of oleoresin samples.

\section{Conclusion}

The choice of a method between the three described in this paper depends on the wanted analysis.

The UV spectrophotometry at $343 \mathrm{~nm}$ is available for a rapid and global determination of pepper oleoresin pungency.

But for a quantitation of only piperine, gas chromatography or high-performance liquid chromatography at $254-280-343$ or $364 \mathrm{~nm}$ can be used and give similar results. However the GC analysis is faster than the HPLC analysis.

The HPLC method permits a good separation of the different alkaloids, but the GC method is more available for the observation of the piperine degradation state because the photoisomers are well separated to the piperine.

\section{References}

1. Lupina, T.; Cripps, H. J. Assoc. Off. Anal. Chem. 1987, 70(1), 112-113.

2. Norme AFNOR NF V 32-078.

3. Archer, A. W. J. Chromatography 1986, 351, 595-598.

4. Weaver, K. M.; Neale, M. E.; Laneville, A. J. Assoc. Off. Anal. Chem. 1988, 71(1), 53-55.

5. Wood, A. B.; Barrow, M. L.; James, D. J. Flavour and Fragance Journal 1988, 3(2), 55-64.

6. Verzele, M.; Van Damme, F.; Schuddinck, G.; Vyncke, P. J. Chromatography 1989, 471, 335-346.

7. De Cleyn, R.; Verzele, M. Chromatographia 1975, 8(7), 342344.

8. Verzele, M.; Redant, G.; Qureshi, P.; Sandra, P. J. Chromatography 1980, 199, 105-122.

9. Glasl, H., Borup-Grochtmann, I.; Wagner, H. Deutsche Apotheker-Zeitung 1976, 116(43), 1638-1641.

10. Norme AFNOR NF X 06-066.

11. Norme AFNOR NF V 03-110.

12. Purseglove, J. W.; Brown, E. G.; Green, C. L.; Robbins, S. R. J. In: Spices. Vol 1. Tropical Agriculture Series Longman, ED. London and Washington 1981; pp 286-439. 